\title{
HADRONIC ASPECTS OF EXOTIC BARYONS
}

\author{
E. OSET, S. SARKAR, M.J. VICENTE VACAS, V. MATEU, \\ Departamento de Física Teórica and IFIC, Centro Mixto Universidad de \\ Valencia-CSIC, Institutos de Investigación de Paterna, Aptd. 22085, 46071 \\ Valencia, Spain \\ T. HYODO, A. HOSAKA \\ Research Center for Nuclear Physics (RCNP), Ibaraki, Osaka 567-0047, Japan \\ F. J. LLANES-ESTRADA \\ Departamento de Fúsica Teórica I, Universidad Complutense, Madrid, Spain \\ In this talk I look into three different topics, addressing first the possibility that \\ the $\Theta^{+}$is a bound state of $K \pi N$, exploiting the results of this study to find \\ out the contribution of two meson and one baryon components in the baryon \\ antidecuplet and in the third place I present results on a new resonant exotic \\ baryonic state which appears as dynamically generated by the Weinberg Tomozawa \\ $\Delta K$ interaction.
}

\section{Is the $\Theta^{+}$a $K \pi N$ bound state?}

The experiment by LEPS collaboration at SPring-8/Osaka ${ }^{1}$ has found a clear signal for an $S=+1$ positive charge resonance around $1540 \mathrm{MeV}$. The signal is also found in many other experiments and not found in some experiments at high energy, and is subject of intense study in different labs to obtain higher statistics. A list of papers on the issue can be found in

2 . At a time when many low energy baryonic resonances are well described as being dynamically generated as meson baryon quasibound states within chiral unitary approaches ${ }^{10,3,4,5,6}$ it looks tempting to investigate the pos- 


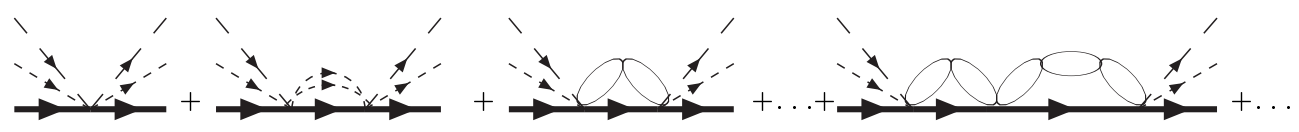

Figure 1. Diagrams considered in the $\kappa N$ interaction.

sibility of this state being a quasibound state of a meson and a baryon or two mesons and a baryon. Its nature as a $K N$ s-wave state is easily ruled out since the interaction is repulsive. $K N$ in a p-wave, which is attractive, is too weak to bind. The next logical possibility is to consider a quasibound state of $K \pi N$, which in s-wave would naturally correspond to spin-parity $1 / 2^{+}$, the quantum numbers suggested in ${ }^{7}$. Such an idea has already been put forward in ${ }^{11}$ where a study of the interaction of the three body system is conducted in the context of chiral quark models. A more detailed work is done in ${ }^{12}$, which we summarize here.

Upon considering the possible structure of $\Theta^{+}$we are guided by the experimental observation ${ }^{8}$ that the state is not produced in the $K^{+} p$ final state. This would rule out the possibility of the $\Theta$ state having isospin $\mathrm{I}=1$. Then we accept the $\Theta^{+}$to be an $\mathrm{I}=0$ state. As we couple a pion and a kaon to the nucleon to form such state, a consequence is that the $K \pi$ substate must combine to $I=1 / 2$ and not $I=3 / 2$. This is also welcome dynamically since the s-wave $K \pi$ interaction in $\mathrm{I}=1 / 2$ is attractive (in $\mathrm{I}=3 / 2$ repulsive) 13. The attractive interaction in $I=1 / 2$ is very strong and gives rise to the dynamical generation of the scalar $\kappa$ resonance around $850 \mathrm{MeV}$ and with a large width ${ }^{13}$.

In order to determine the possible $\Theta^{+}$state we search for poles of the $K \pi N \rightarrow K \pi N$ scattering matrix. To such point we construct the series of diagrams of fig. 1, where we account explicitly for the $K \pi$ interaction by constructing correlated $K \pi$ pairs and letting the intermediate $K \pi$ and nucleon propagate. This requires a kernel for the two meson-nucleon interaction which we now address. We formulate the meson-baryon lagrangian in terms of the $\mathrm{SU}(3)$ matrices, $B, \Gamma_{\mu}, u_{\mu}$ and the implicit meson matrix 
$\Phi$ standard in $\mathrm{ChPT}^{9}$,

$L=\operatorname{Tr}\left(\bar{B} i \gamma^{\mu} \nabla_{\mu} B\right)-M_{B} \operatorname{Tr}(\bar{B} B)+\frac{1}{2} D \operatorname{Tr}\left(\bar{B} \gamma^{\mu} \gamma_{5}\left\{u_{\mu}, B\right\}\right)+\frac{1}{2} F \operatorname{Tr}\left(\bar{B} \gamma^{\mu} \gamma_{5}\left[u_{\mu}, B\right]\right)$

with the definitions in ${ }^{9}$.

First there is a contact three body force simultaneously involving the pion, kaon and nucleon, which can be derived from the meson- baryon Lagrangian term containing the covariant derivative $\nabla_{\mu}$.

By taking the isospin $\mathrm{I}=1 / 2 \kappa$ states and combining them with the nucleon we generate $\mathrm{I}=0,1$ states which diagonalize the scattering matrix associated to $t_{m B}$ and we find that the interaction in the $\mathrm{I}=0$ channel is attractive, while in the $\mathrm{I}=1$ channel is repulsive. This would give chances to the $\kappa N t$-matrix to develop a pole in the bound region, but rules out the $\mathrm{I}=1$ state.

The series of terms of Fig. 1 might lead to a bound state of $\kappa N$ which would not decay since the only intermediate channel is made out of $K \pi N$ with mass above the available energy. The decay into $K N$ observed experimentally can be taken into account explicitly and this and other diagrams accounting for the interaction of the mesons with the other meson or the nucleon are taken into account in the calculations ${ }^{12}$.

What we find at the end is that, in spite of the attraction found, this interaction is not enough to bind the system, since we do not find a pole below the $K \pi N$ threshold. In order to quantify this second statement we increase artificially the potential $t_{m B}$ by adding to it a quantity which leads to a pole around $\sqrt{s}=1540 \mathrm{MeV}$ with a width of around $\Gamma=40 \mathrm{MeV}$. This is accomplished by adding an attractive potential around five or six times bigger than the existing one. We should however note that we have not exhausted all possible sources of three body interaction since only those tied to the Weinberg Tomozawa term have been considered. We think that some more work in this direction should be still encouraged and there are already some steps given in ${ }^{14}$. 


\section{Coupling of the $\Theta^{+}$to $K \pi N$}

Although not enough to bind the $K \pi N$ system, the interaction has proved attractive in $\mathrm{L}=0$ and $\mathrm{I}=0$. This, together with the proximity of the $\Theta^{+}$ mass to the $K \pi N$ threshold ( $30 \mathrm{MeV}$ ) suggests that the $\Theta^{+}$should have a non negligible $K \pi N$ component in its wave function. The procedure followed in ${ }^{15}$ to find out the contribution to the binding is the following: 1 ) one assumes that the $\Theta^{+}$belongs to the standard antidecuplet of baryons suggested in ${ }^{7}$. 2) The $N^{*}(1710)$ is assumed to have a large component corresponding to this antidecuplet. 3) From the large decay of the $N^{*}(1710)$ into $\pi \pi N$, both in s-wave and p-wave, we extract the strength for two $\mathrm{SU}(3)$ invariant phenomenological potentials which allow us to extend the coupling to different meson meson baryon components of all baryons of the antidecuplet. 4) A selfenergy diagram is constructed with two vertices from these Lagrangians and two meson and a baryon intermediate states. 5) Regularization of the loops is done with a cut off similar to the one needed in the study of the $\bar{K} N$ interaction ${ }^{3}$ and this leads to attractive selfenergies of the order of 100-150 MeV. At the same time one finds an energy splitting between the different members of the antidecuplet of the order of $20 \mathrm{MeV}$, or 20 percent of the empirical values, with the right ordering demanded by the Gell-Mann-Okubo rule, and hence a maximum binding for the $\Theta^{+}$.

This finding means that detailed studies of the $\Theta^{+}$should take into consideration this important component of $K \pi N$ which helps produce extra binding for the $\Theta^{+}$, one of the problems faced by ordinary quark models.

The finding of this work has repercussions in the selfenergy of the $\Theta^{+}$ in nuclei. Indeed, as found in ${ }^{16}$, when one takes into account the pionic medium polarization, exciting $p h$ and $\Delta h$ components with the pion, the mechanism leads to an extra attraction in the medium which is of the order of $50-100 \mathrm{MeV}$ at normal nuclear matter density. This, together with the other finding of a very small imaginary part of the selfenergy, leads to levels of the $\Theta^{+}$which are separated by energies far larger than the width 
of the states. This makes it a clear case for experimental observation and suggestions of reactions have already been made ${ }^{17}$.

\section{A resonant $\Delta K$ state as a dynamically generated exotic baryon}

Given the success of the chiral unitary approach in generating dynamically low energy resonances from the interaction of the octets of stable baryons and the pseudoscalar mesons, in ${ }^{18}$ the interaction of the decuplet of $3 / 2^{+}$ with the octet of pseudoscalar mesons was studied and shown to lead to many states which were associated to experimentally well established resonances. The purpose of the present work is to show that this interaction leads also to a new state of positive strangeness, with $I=1$ and $J^{P}=3 / 2^{-}$, hence, an exotic baryon described in terms of a resonant state of a $\Delta$ and a $K$.

The lowest order chiral Lagrangian for the interaction of the baryon decuplet with the octet of pseudoscalar mesons is given by 19

$$
L=i \bar{T}^{\mu} D_{\nu} \gamma^{\nu} T_{\mu}-m_{T} \bar{T}^{\mu} T_{\mu}
$$

where $T_{a b c}^{\mu}$ is the spin decuplet field and $D^{\nu}$ the covariant derivative given in ${ }^{19}$. The identification of the physical decuplet states with the $T_{a b c}^{\mu}$ can be seen in ${ }^{20}$.

For strangeness $S=1$ and charge $Q=3$ there is only one channel $\Delta^{++} K^{+}$which has $I=2$. For $S=1$ and $Q=2$ there are two channels $\Delta^{++} K^{0}$ and $\Delta^{+} K^{+}$. From these one can extract the transition amplitudes for the $I=2$ and $I=1$ combinations and we find ${ }^{20}$

$$
V(S=1, I=2)=\frac{3}{4 f^{2}}\left(k^{0}+k^{0}\right) ; \quad V(S=1, I=1)=-\frac{1}{4 f^{2}}\left(k^{0}+k^{\prime 0}\right),
$$

where $k\left(k^{\prime}\right)$ indicate the incoming (outgoing) meson momenta. These results indicate that the interaction in the $I=2$ channel is repulsive while it is attractive in $I=1$. 
The use of $V$ as the kernel of the Bethe Salpeter equation ${ }^{3}$, or the N/D unitary approach of ${ }^{4}$ both lead to the scattering amplitude

$$
t=(1-V G)^{-1} V
$$

In eq. (4), $V$ factorizes on shell ${ }^{3,4}$ and $G$ stands for the loop function of the meson and baryon propagators, the expressions for those being given in ${ }^{3}$ for a cut off regularization and in ${ }^{4}$ for dimensional regularization.

Next we fix the scale of regularization by determining the cut off, $q_{\max }$, in the loop function of the meson and baryon propagators in order to reproduce the resonances for other strangeness and isospin channels. They are one resonance in $(I, S)=(0,-3)$, another one in $(I, S)=(1 / 2,-2)$ and another one in $(I, S)=(1,-1)$. The last two appear in 18 around 1800 $\mathrm{MeV}$ and $1600 \mathrm{MeV}$ and they are identified with the $\Xi(1820)$ and $\Sigma(1670)$. We obtain the same results as in ${ }^{18}$ using a cut off $q_{\max }=700 \mathrm{MeV}$.

With this cut off we explore the analytical properties of the amplitude for $S=1, I=1$ in the first and second Riemann sheets. First we see that there is no pole in the first Riemann sheet.

Next we explore the second Riemann sheet which we obtain by changing the sign of the momentum in the expresssion for the meson baryon loop function.

We find a pole at $\sqrt{s}=1635 \mathrm{MeV}$ in the second Riemann sheet.

The situation in the scattering matrix is revealed in fig. 2 which shows the real and imaginary part of the $K \Delta$ amplitudes for the case of $I=1$. For the case of $I=2$ the imaginary part follows the ordinary behaviour of the opening of a threshold, growing smoothly from threshold. The real part is also smooth. For the case of $I=1$, instead, the strength of the imaginary part is stuck to threshold as a reminder of the existing pole in the complex plane, growing very fast with energy close to threshold. The real part has also a pronounced cusp at threshold, which is also tied to the same singularity. 


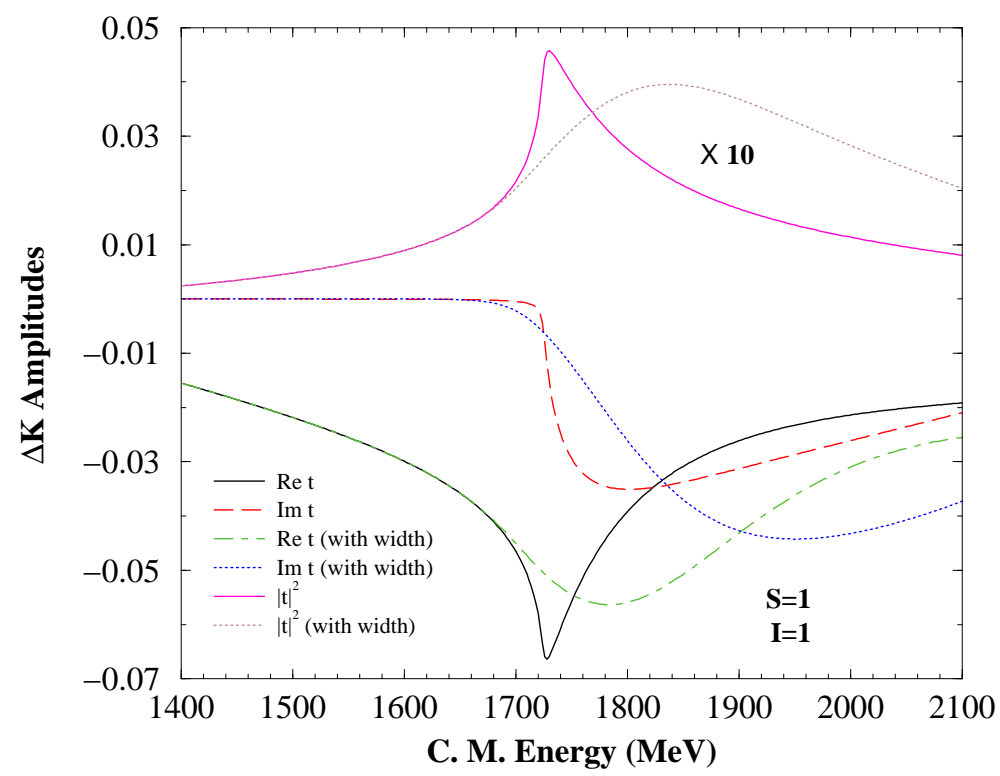

Figure 2. Amplitudes for $\Delta K \rightarrow \Delta K$ for $I=1$

In figure 2 and we see that the peak around threshold becomes smoother and some strength is moved to higher energies when we consider the width of the $\Delta$ in the intermediate states. Even then, the strength of the real and imaginary parts in the $I=1$ are much larger than for $I=2$. The modulus squared of the amplitudes shows some peak behavior around $1800 \mathrm{MeV}$ in the case of $I=1$, while it is small and has no structure in the case of $I=2$.

We propose the study of the following reactions: 1) $\left.p p \rightarrow \Lambda \Delta^{+} K^{+}, 2\right)$ $\left.p p \rightarrow \Sigma^{-} \Delta^{++} K^{+}, 3\right) p p \rightarrow \Sigma^{0} \Delta^{++} K^{0}$. In the first case the $\Delta^{+} K^{+}$state produced has necessarily $I=1$. In the second case the $\Delta^{++} K^{+}$state has $I=2$. In the third case the $\Delta^{++} K^{0}$ state has mostly an $I=1$ component. The experimental confirmation of the results found here through the study of the $\Delta K$ invariant mass distribution in these reactions would give support to this new exotic baryonic state which stands as a resonant $\Delta K$ state. 


\section{Acknowledgments}

This work is partly supported by DGICYT contract number BFM200300856, the E.U. EURIDICE network contract no. HPRN-CT-2002-00311 and the Research Cooperation program of the JSPS and the CSIC.

\section{References}

1. T. Nakano et al. [LEPS Collaboration], Phys. Rev. Lett. 91 (2003) 012002.

2. T. Hyodo, http://www.rcnp.osaka-u.ac.jp/ hyodo/research/Thetapub.html

3. E. Oset and A. Ramos, Nucl. Phys. A 635 (1998) 99.

4. J. A. Oller and U. G. Meissner, Phys. Lett. B 500 (2001) 263.

5. D. Jido, J. A. Oller, E. Oset, A. Ramos and U. G. Meissner, Nucl. Phys. A 725 (2003) 181.

6. J. Nieves and E. Ruiz Arriola, Phys. Rev. D 64 (2001) 116008.

7. D. Diakonov, V. Petrov and M.V. Polyakov, Z. Phys. A359 (1997) 305.

8. J. Barth et al. [SAPHIR Collaboration], Phys. Lett. B 572 (2003) 127.

9. V. Bernard, N. Kaiser and U.G. Meissner, Int. J. Mod. Phys. E4 (1995) 193.

10. N. Kaiser, P. B. Siegel and W. Weise, Nucl. Phys. A 594 (1995) 325.

11. P. Bicudo and G. M. Marques, Phys. Rev. D 69 (2004) 011503.

12. F. J. Llanes-Estrada, E. Oset and V. Mateu, Phys. Rev. C 69 (2004) 055203.

13. J. A. Oller, E. Oset and J. R. Pelaez, Phys. Rev. D 59 (1999) 074001 [Erratum-ibid. D 60 (1999) 099906].

14. T. Kishimoto and T. Sato, arXiv:hep-ex/0312003.

15. T. Hyodo, F. Llanes, E. Oset, A. Hosaka, J.R. Peláez, M.J. Vicente Vacas, to be submitted.

16. D. Cabrera, Q. B. Li, V. K. Magas, E. Oset and M. J. Vicente Vacas, arXiv:nucl-th/0407007.

17. H. Nagahiro, S. Hirenzaki, E. Oset and M. J. Vicente Vacas, arXiv:nuclth/0408002.

18. E. E. Kolomeitsev and M. F. M. Lutz, Phys. Lett. B 585, 243 (2004).

19. E. Jenkins and A. V. Manohar, Phys. Lett. B 259 (1991) 353.

20. S. Sarkar, E. Oset and M. J. Vicente Vacas, arXiv:nucl-th/0404023. 